Review

\title{
Natural Compounds from Saffron and Bear Bile Prevent Vision Loss and Retinal Degeneration
}

\author{
Laura Fernández-Sánchez ${ }^{1, \dagger}$, Pedro Lax ${ }^{1, \dagger}$, Agustina Noailles ${ }^{1}$, Antonia Angulo ${ }^{2}$, \\ Victoria Maneu ${ }^{2}$ and Nicolás Cuenca ${ }^{1, *}$
}

1 Departament of Physiology, Genetics and Microbiology, University of Alicante, 03690 Alicante, Spain; E-Mails: laura.fs@ua.es (L.F.-S.); pedro.lax@ua.es (P.L.); noailles24@gmail.com (A.N.)

2 Department of Optics, Pharmacology and Anatomy, University of Alicante, 03690 Alicante, Spain; E-Mails: angulo@ua.es (A.A.); vmaneu@ua.es (V.M.)

$\dagger$ These authors contributed equally to this work.

* Author to whom correspondence should be addressed; E-Mail: cuenca@ua.es; Tel.: +34-96-590-9916; Fax: +34-96-590-9569.

Academic Editor: Peter Koulen

Received: 19 June 2015 / Accepted: 28 July 2015 / Published: 31 July 2015

\begin{abstract}
All retinal disorders, regardless of their aetiology, involve the activation of oxidative stress and apoptosis pathways. The administration of neuroprotective factors is crucial in all phases of the pathology, even when vision has been completely lost. The retina is one of the most susceptible tissues to reactive oxygen species damage. On the other hand, proper development and functioning of the retina requires a precise balance between the processes of proliferation, differentiation and programmed cell death. The life-or-death decision seems to be the result of a complex balance between pro- and anti-apoptotic signals. It has been recently shown the efficacy of natural products to slow retinal degenerative process through different pathways. In this review, we assess the neuroprotective effect of two compounds used in the ancient pharmacopoeia. On one hand, it has been demonstrated that administration of the saffron constituent safranal to $\mathrm{P} 23 \mathrm{H}$ rats, an animal model of retinitis pigmentosa, preserves photoreceptor morphology and number, the capillary network and the visual response. On the other hand, it has been shown that systemic administration of tauroursodeoxycholic acid (TUDCA), the major component of bear bile, to $\mathrm{P} 23 \mathrm{H}$ rats preserves cone and rod structure and function, together with their contact with postsynaptic neurons. The neuroprotective effects of safranal and TUDCA make these compounds potentially useful for therapeutic applications in retinal degenerative diseases.
\end{abstract}


Keywords: retina; apoptosis; oxidative stress; Crocus sativus; safranal; tauroursodeoxycholic acid; TUDCA; P23H

\section{Introduction}

The retina is the light-sensitive tissue that lines the inner surface of the eye and is in charge of the first steps of visual processing. The structural and functional complexity of the retina makes this tissue vulnerable to alterations from any sort of pathological injury. At the cellular and molecular level, the response of the retina to injury is similar in all retinal neurodegenerative diseases, and results in a set of cell signals that lead to well-established and similar morphological and functional impairments. Regardless of the aetiology of the damage, all retinal disorders involve the activation of oxidative stress and apoptosis pathways [1].

The retina is one of the most susceptible tissues to reactive oxygen species (ROS) damage. Photoreceptor cells are continuously exposed to light, and are one of the highest consumers of oxygen in the central nervous system (CNS). In this context, ellipsoids of photoreceptors exhibit a high density of mitochondria [2] that provide energy for phototransduction and the maintenance of $\mathrm{Ca}^{2+}$ homeostasis [3]. Moreover, experimental evidences show the presence of aerobic metabolism in the membranous disks of photoreceptor outer segments $[4,5]$. The energy required for synaptic vesicular trafficking and regulation of the cytosolic $\mathrm{Ca}^{2+}$ levels in the presynaptic terminals of photoreceptors is provided by mitochondria present in both rod spherules and cone pedicles [6]. Dysfunctional mitochondria cause an energy deficit, leading to an increase of ROS levels and an abnormal elevation of cytosolic $\mathrm{Ca}^{2+}[7,8]$. It is widely accepted that oxidative stress plays a central role in retinal degeneration. Thus, for example, oxidative stress associated with smoking has been considered one of the most important risk factors in the development of age-related macular degeneration (AMD) [9,10]. In retinitis pigmentosa (RP) and glaucoma, the apoptotic stimuli are also exacerbated by oxidative stress [11-13]. In diabetic retinopathy (DR), hyperglycemia drives mitochondria to increase their activity [14,15], which results in an overproduction of superoxide from mitochondria [16-18].

Proper development and functioning of the retina requires a precise balance between the processes of proliferation, differentiation and programmed cell death. Most defective, unwanted and potentially dangerous cells die by apoptosis, a controlled genetic program for removing cells without damaging the surrounding tissue [19]. The life-or-death decision seems to be the result of a complex balance between pro- and anti-apoptotic signals [20-22]. The progressive loss of vision via photoreceptor cell death is a shared trait of all inherited retinal diseases [22,23]. The types of cell death within the affected retina are variable, with different or multiple forms of cell death discovered in different models of the same disease [23-25]. In human samples and animal models of retinitis pigmentosa, the morphological characteristics of apoptosis have been described [26-28], even though more recent evidences suggest that photoreceptor cell death may result primarily from non-apoptotic mechanisms [23-25].

Retinitis pigmentosa constitutes a large heterogeneous group of inherited neurodegenerative retinal disorders that cause a progressive loss of retinal function and represent a major cause of blindness. More than 100 different mutations in the rhodopsin-encoding gene (RHO) are associated with RP, together 
accounting for $30 \%$ to $40 \%$ of autosomal dominant RP (adRP) cases [29]. The P23H mutation in RHO is the most prevalent cause of adRP [30], which alone accounts for approximately $12 \%$ of adRP cases in the United States [31]. The majority of RP-causing mutations in the RHO gene, including P23H, cause misfolding and retention of rhodopsin in the endoplasmic reticulum [32]. These studies also suggest that the mechanism of RP involves a cellular stress response [33], the final common pathway being programmed during photoreceptor cell death [34]. Transgenic $\mathrm{P} 23 \mathrm{H}$ rats mimic the clinical findings reported for human patients with P23H RP [35,36]. These animals develop a progressive rod dysfunction, albeit initially exhibiting a normal cone function. The loss of photoreceptors is accompanied by degenerative changes in the inner retina [37], including a substantial degeneration of retinal ganglion cells [38-40]. P23H line 3 rats retain vision for relatively long periods of their lives, similarly to findings in $\mathrm{P} 23 \mathrm{H}$ humans, who exhibit significantly better visual acuity and greater ERG amplitudes than patients harboring other RP mutations [35,36].

Neuroprotective treatments provide therapeutic strategies independent of the aetiology of the degeneration. The aim of neuroprotective mechanisms is to provide an adequate environment in which to prolong the viability of retinal cells through their effects on a number of biochemical pathways. This can be achieved by either delivering neurotrophic growth factors to retinal tissues, inhibiting pro-apoptotic pathways or implementing viability factors. In this context, traditional medicine provides frontline pharmacotherapy for many millions of people worldwide. In this review, we will discuss the efficacy of two natural compounds, safranal and TUDCA, to slow retinal degenerative process through different pathways. The administration of neuroprotective factors, which slow retinal degeneration, may be crucial in all phases of the pathology, even when vision has been completely lost.

\section{Results and Discussion}

Human retinal degenerative diseases are currently incurable and retinal degeneration, once initiated, is irreversible. The therapies applied at present in the treatment of retinal dystrophies are focused on delaying the onset or progression of degeneration, but no therapies are available to replace lost retinal cells or restore accurate vision. Currently, the potential therapeutic approaches aimed at finding a cure for blinding diseases focus on two main lines of action. First is the use of preventive strategies that attempt to counteract the underlying disease mechanisms, either by manipulating cellular pathways through the use of pharmacological compounds or genetic modification by gene silencing and/or gene replacement. The second approach is not concerned as much with the causes of the diseases as it is with ways to prevent cell death, such as the administration of anti-apoptotic, anti-inflammatory and neurotrophic compounds. In this context, the administration of antioxidants (alone or in cocktails), anti-apoptotics, anti-inflammatories, neurotrophic factors or viability factors may slow the neurodegeneration of the retina by delaying retinal cell death [41-44].

\subsection{Saffron Constituents Prevent Vision Loss and Retinal Degeneration}

The pistil of Crocus sativus, commonly known as saffron, has been commonly used in traditional medicine as an anodyne, sedative and emmenagogue. In traditional Islamic medicine, they are attributed a wide range of activities: such as oxytocic, anti-carcinogenic, exhilarant, anti-depressant, and anti-asthma 
effects $[45,46]$. More recently, the active constituents of saffron (crocetin, crocin and safranal) have been described as powerful carotenoid antioxidants with protective and therapeutic properties [47-49].

Extracts from saffron have been used to delay retinal degeneration in early stages of age-related macular degeneration [50] and in a rodent model of light-induced retinal damage [51]. In albino rats fed on saffron supplements, the effects of continuous bright light exposure were significantly diminished, and the morphology and function of the retina were maintained [51-53]. Additionally, in clinical trials involving human patients with early AMD, $20 \mathrm{mg}$ per day of saffron supplementation for 90 days significantly improved some parameters of the macular photopic flash electroretinogram, such as amplitude and modulation threshold [50].

Crocetin is one of the major active compounds in saffron [54]. Crocetin has been reported to prevent retinal degeneration induced by oxidative and endoplasmic reticulum stresses via the inhibition of caspase 3 and 9 activities in the RGC-5 retinal ganglion cell line in vitro and in a mouse model of light-induced retinal degeneration (LIRD) in vivo [55]. Moreover, crocetin inhibited retinal ischemic damage in mice, preventing the apoptotic death of ganglion cells and the reduction of the inner nuclear layer (INL) by decreasing the activation of mitogen-activated protein kinases $(\mathrm{p} 38, \mathrm{JNK})$ and redox-sensitive transcription factors (NF-kB and c-Jun), while maintaining the functional activity of the retina [56]. Crocetin also prevented NMDA-induced murine retinal damage by inhibiting both caspases 3 and 7 activation and the increased expression of cleaved caspase 3 in the ganglion cell layer (GCL) and INL [57].

The carotenoid crocin is one of the constituents of saffron stigmas [54]. Treatment with crocin protected retinal photoreceptors against light-induced cell death in primary cell cultures from primate and bovine retinas [58]. Cell death was significantly attenuated in cell cultures pretreated with various concentrations of crocin. On the other hand, crocin analogs were found to increase the blood flow in the retina and choroid and to facilitate retinal function recovery [59]. In rat models of retinal ischemia/reperfusion, crocin prevented injury-induced apoptosis of RGCs by activating the PI3K/AKT signaling pathway [60].

Safranal has been seen to prevent both the decrease of antioxidant enzyme activities and lipid peroxidation occurring with age in rat livers [61]. In ischemic rats, safranal also exerted a protective action against oxidative damage in skeletal muscle [62] and cerebral tissues [63]. Administration of the saffron constituent safranal to $\mathrm{P} 23 \mathrm{H}$ rats, an animal model of retinitis pigmentosa, preserved photoreceptor morphology and number, and the visual response (Figure 1) [42]. The analysis of the capillary network revealed that safranal is also able to prevent the loss of retinal vessels that occurs in the $\mathrm{P} 23 \mathrm{H}$ rat retina [42], evidencing more extensive capillary networks and better-preserved capillary loops, as compared to untreated animals. These evidences suggest that the neuroprotective action of safranal extends not only to photoreceptor cells, but also to other retinal cells.

\subsection{Bear Bile Constituents Prevent Vision Loss and Retinal Degeneration}

Bear bile has been used in traditional Chinese medicine for over 3000 years to treat visual disorders [64]. Tauroursodeoxycholic acid (TUDCA), the major component of bear bile, has been shown to exhibit antiapoptotic properties in neurodegenerative diseases, including those affecting the retina. Systemic administration of TUDCA has been demonstrated to slow retinal degeneration in both the $\operatorname{rd} 10$ autosomal recessive RP mouse model [64-68] and in a LIRD mouse model [67]. In these two retinal 
degeneration models, TUDCA-treated animals were shown to maintain better visual function, thicker outer nuclear layer (ONL) and better preservation of outer segments than untreated animals. TUDCA also prevented retinal degeneration in the $\mathrm{P} 23 \mathrm{H}$ autosomal dominant $\mathrm{RP}$ rat model (Figure 1) [41]. P23H treated rats showed higher a- and b-wave amplitudes under both photopic and scotopic conditions than untreated rats. Moreover, TUDCA decreased photoreceptor apoptosis and maintained synaptic connectivity among retinal cells [41]. Furthermore, $\mathrm{P} 23 \mathrm{H}$ rats treated with TUDCA exhibited greater labeling for cytochrome c oxidase subunit IV in photoreceptor cell mitochondria, which is closely related to improved physiological activity and oxidative metabolism [6].

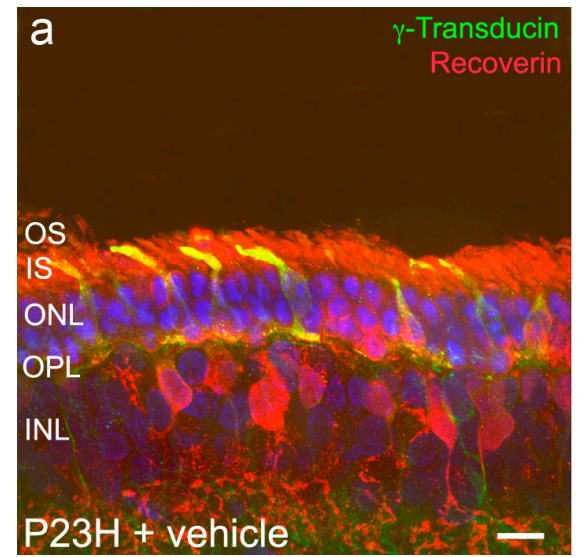

d $\mathrm{P} 23 \mathrm{H}+$ vehicle
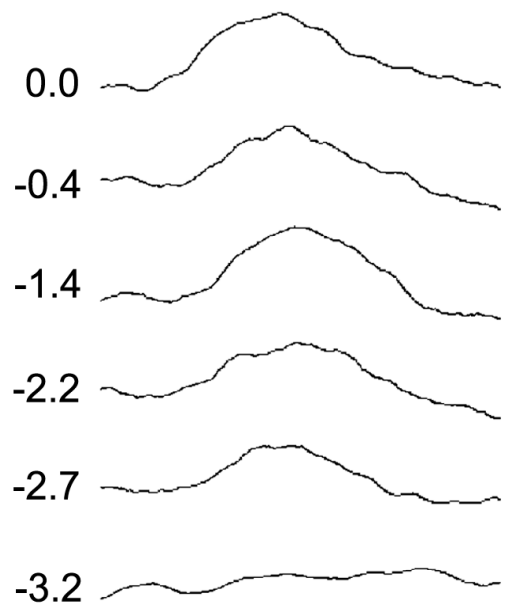

$-3.8$

$-4.5$

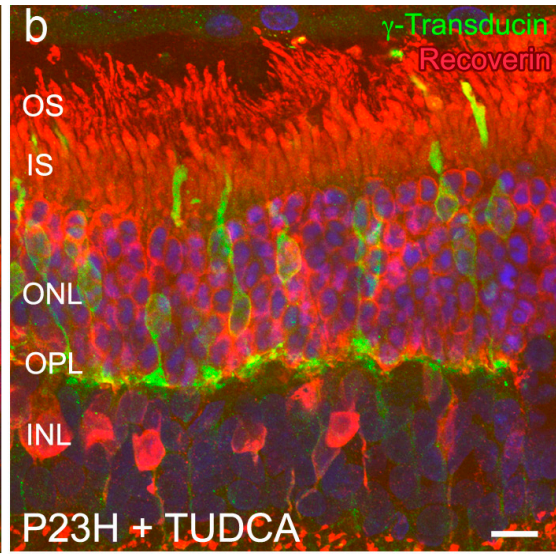

e $\mathrm{P} 23 \mathrm{H}+\mathrm{TUDCA}$

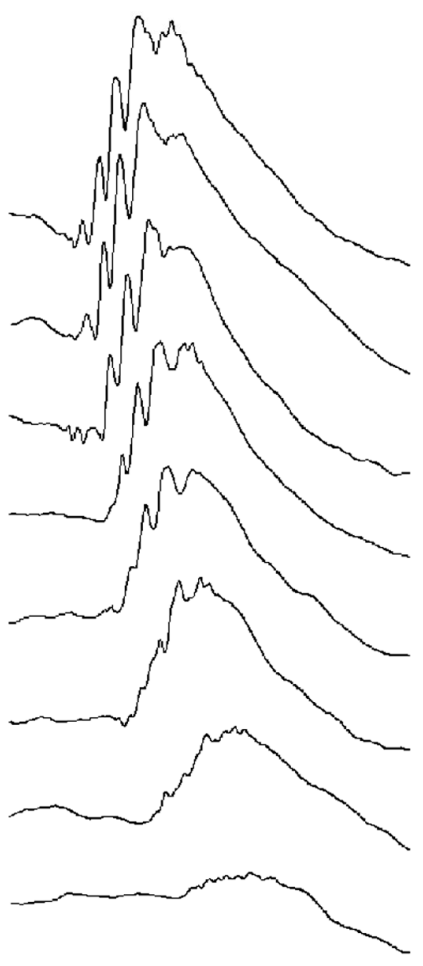



f $\mathrm{P} 23 \mathrm{H}+$ safranal

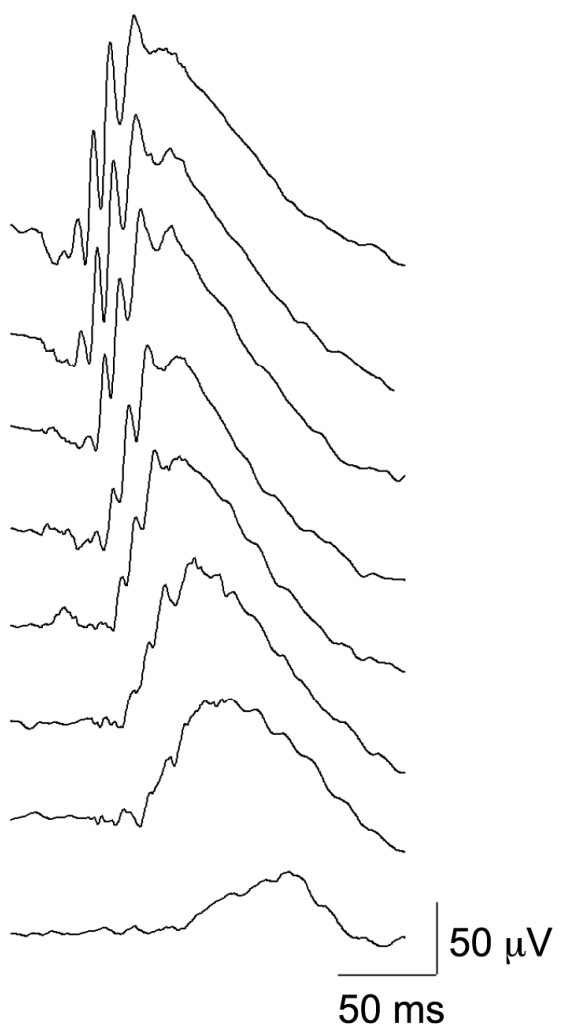

Figure 1. Cont. 

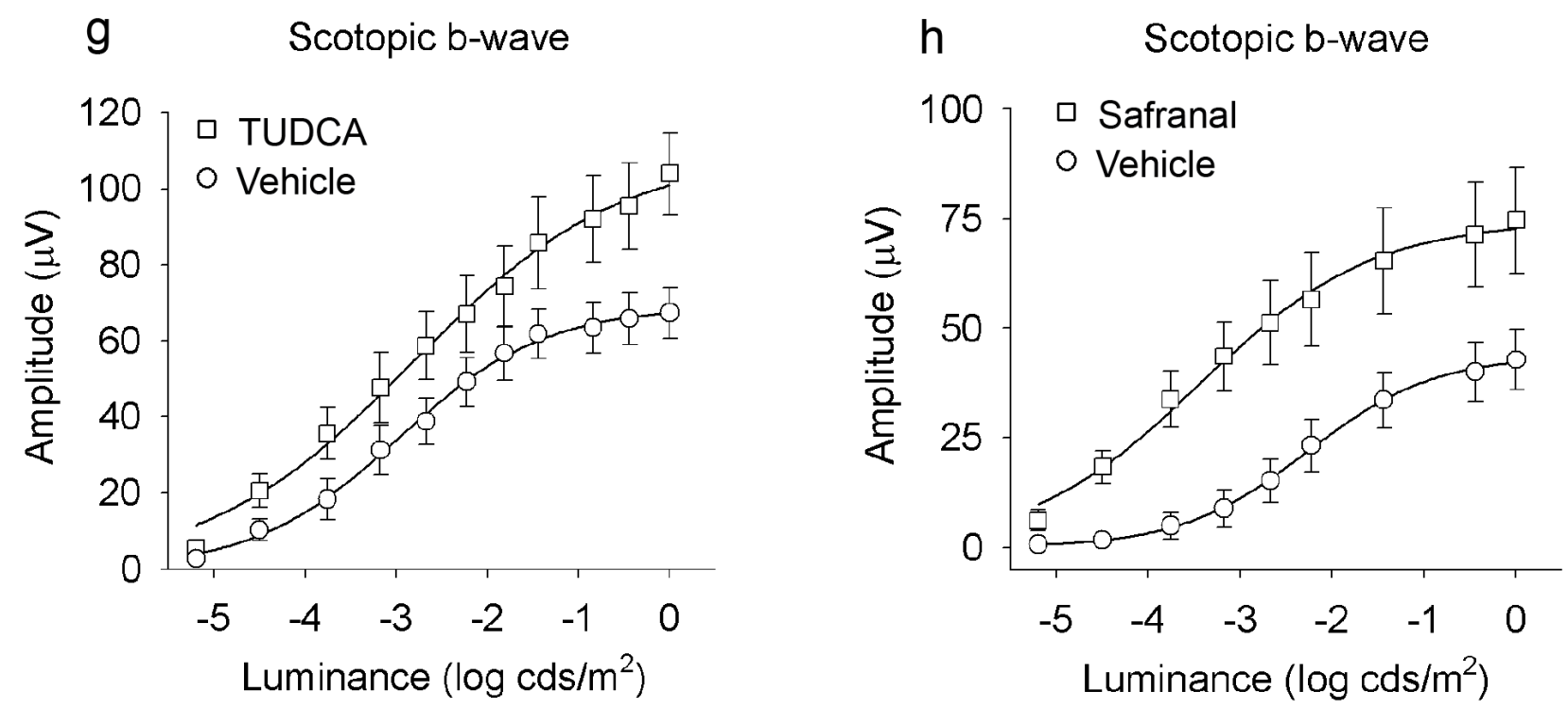

Figure 1. Neuroprotective effects of TUDCA and safranal on the morphological and functional changes associated to retinal degeneration. $(\mathbf{a}-\mathbf{c})$ Immunolabeling of retinal vertical sections for $\gamma$-transducin (cones, green) and recoverin (rods, cones, and two bipolar cell subtypes, red) in P120 P23H rats treated with vehicle (a) TUDCA (b) or safranal (c). Nuclei stained with TO-PRO 3 (blue). Images were collected from the central area of the retina, close to the optic nerve; (d-f) Representative scotopic full-field ERG waveforms from P120 P23H rats treated with vehicle (d) TUDCA (e) or safranal (f). Units on the left indicate input flash intensities in $\log \mathrm{cd} \cdot \mathrm{s} / \mathrm{m}^{2}$. Note that ERG amplitudes in the $\mathrm{P} 23 \mathrm{H}$ rat treated with TUDCA of safranal are higher than those recorded in the vehicle-treated animal; (g-h) Stimulus intensity curves for mixed scotopic b-waves from rats administered with TUDCA (g, squares), safranal (h, squares) or vehicle (g-h, circles). OS: outer segments, IS: inner segments, ONL: outer nuclear layer, OPL: outer plexiform layer, INL: inner nuclear layer. Scale bars: $10 \mu \mathrm{m}$.

In addition to its anti-apoptotic properties, TUDCA has also been shown to exert anti-inflammatory, antioxidant and chaperone activities. In this context, TUDCA suppressed the formation of laser-induced choroidal neovascularization (CNV) in rats by decreasing the number and size of CNV lesions, probably due to its anti-inflammatory properties, which diminished vascular endothelial growth factor (VEGF) levels in the retina after the laser treatment [69]. Additionally, systemic administration of TUDCA preserved photoreceptors after retinal detachment in rats, preventing the reduction in ONL thickness, and this was accompanied by decreased oxidative stress and inhibition of the increase in caspase 3 and 9 activity [70]. TUDCA also protected retinal neural cell cultures from high glucose-induced death by decreasing mitochondrial-nuclear translocation of the apoptosis inducing factor (AIF). This inhibition of the release of AIF from the mitochondria was probably due to the antioxidant properties of TUDCA, as corroborated by the marked decrease in oxidative stress biomarkers with TUDCA treatment [71]. These findings may have relevance in the treatment of DR. Furthermore, systemic injection of TUDCA diminished endoplasmic reticulum stress, prevented apoptosis and reduced cone degeneration in the retina of a mouse model of Leber congenital amaurosis [72]. 


\subsection{TUDCA Reduces Microglial Cell Activation in Degenerative Retinas}

Microglial cells in the retina act as sensors of disarrangement in their microenvironment. Their balanced activities play a key role in the survival of neurons [73,74]. Activation of the microglia has been demonstrated in association with several neurodegenerative diseases, such as Alzheimer's and Parkinson's diseases, amyotrophic lateral sclerosis, and multiple sclerosis, although it remains unclear whether microglial activation is a cause or a consequence of neuronal damage [75-79].

Changes in microglial cells number, activation and distribution have been reported in different forms of disease or retinal damage, like glaucoma [80,81], age-related macular degeneration [82,83], light damage $[84,85]$ and retinitis pigmentosa [86]. In $\mathrm{P} 23 \mathrm{H}$ rat retinas, microglia density increased in the GCL, IPL (inner plexiform layer) and OPL (outer plexiform layer), microglial cells appears in the subretinal space (SS), and a great deal of microglial cells were labeled with anti-MHC-II, a marker of microglia activation (Figure 2). In the mouse model of retinitis pigmentosa rd10, it has been demonstrated high levels of pro-inflammatory cytokines and chemokines and early microglia activation [87,88]. Previous studies have also demonstrated increased density of macrophages after microglia activation [89].

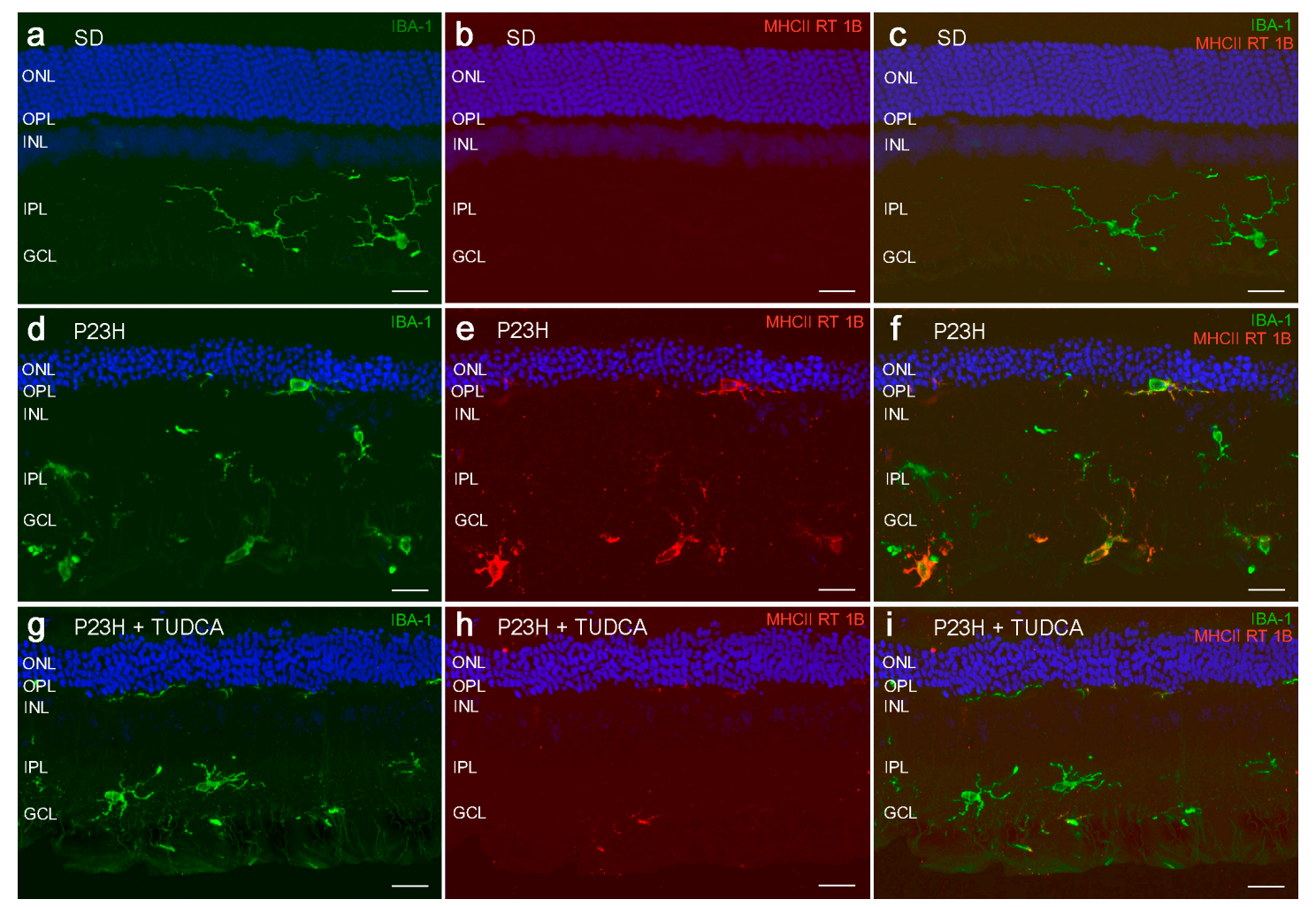

Figure 2. Activation of microglial cells. Vertical sections of retinas from a SD (a-c), untreated $\mathrm{P} 23 \mathrm{H}(\mathbf{d}-\mathbf{f})$ and TUDCA-treated $\mathrm{P} 23 \mathrm{H}(\mathbf{g}-\mathbf{i})$ rat at $\mathrm{P} 120$ stained for Ibal (green; $\mathbf{a}, \mathbf{d}, \mathbf{g})$ MHC-II RT 1B (red; b, e, h) or both (c, f, i). Nuclei stained with TO-PRO 3 (blue). All images were collected from the central area of the retina, close to the optic nerve. Note that microglia density in $\mathrm{P} 23 \mathrm{H}$ rats treated with TUDCA is similar to the one observed in the SD rats and smaller than that shown in untreated $\mathrm{P} 23 \mathrm{H}$ rats. The relative number of MHC-II-positive cells in TUDCA-treated P23H rats is also low. ONL: outer nuclear layer, OPL: outer plexiform layer, INL: inner nuclear layer, IPL: inner plexiform layer, GCL: ganglion cell layer. Scale bars: $20 \mu \mathrm{m}$. 
Systemic administration of TUDCA reduced the number and activation of microglial cells in $\mathrm{P} 23 \mathrm{H}$ rats. Moreover, in TUDCA-treated rat retinas microglia was mainly distributed in more internal retinal layers, GCL and IPL, and they are scarce in the OPL and missing in the SS, similar to that found in normal rat retinas (Figure 2). Attenuation of microglial activation using TUDCA has been also demonstrated in experimental models of neuroinflammation, in which it has been reported that TUDCA reduces in vitro microglial migration and the expression of chemoattractants required for microglial migration [90]. The effects of TUDCA on retinal microglial cells could be also attributed to an effect of TUDCA on microglial cells behavior, presumably interfering in the respiratory burst of the microglia, which is a critical step in its activation $[71,91]$.

\subsection{Neuroprotective Mechanisms}

In RP, the high diversity of genes and mutations involved leads to the activation of a variety of apoptotic and non-apoptotic pathways [23,24,28]. In rodent models of RP, endoplasmic reticulum (ER) stress triggers an increase in cytosolic $\mathrm{Ca}^{2+}$ levels with ensuing up-regulation of caspase 12 [92], which in turn activates caspase 3 . In the $\mathrm{P} 23 \mathrm{H}$ rat retina, rod photoreceptor cells have been shown to die due to apoptosis triggered by ER stress, mainly through caspase 12 [93], although activation of alternative pathways could play a significant role in this process [23-25]. The accumulation of misfolded proteins and increased cytosolic $\mathrm{Ca}^{2+}$ activates additional pro-apoptotic factors that reinforce each other during the apoptosis process (Figure 3) [94]. It has been shown that, besides the activation of caspase 12, ER stress and the subsequent $\mathrm{Ca}^{2+}$ release can activate calcium-dependent cysteine proteases known as calpains $[95,96]$. These enzymes are present in the cytosol, and together with caspase 12 [97] and other pro-apoptotic proteins [96], may amplify the death signal. The activation of calpains has been related to various retinal diseases, and is considered to be one of the most important caspase-independent apoptotic pathways in photoreceptor cell death associated with RP [11,28,98,99]. In this scenario, mitochondria play an important role in apoptosis, due to their rich content in pro-apoptotic proteins (Figure 3) [100]. $\mathrm{Bcl}-2$ is the best-characterized protein family involved in the progression of apoptosis in photoreceptor cells. A decrease in the ratio of Bcl-XL to Bax (Bcl-2-associated X protein) has been evidenced in RP animal models carrying mutations in the rhodopsin gene, thus indicating the implication of mitochondria in the progress of apoptosis [93,101]. In this context, preserving the integrity of the mitochondrial membrane by preventing the formation of mitochondrial outer membrane pores (MOMP) and the modulation of existing mitochondrial channels, such as the mitochondrial permeability transition pore complex, could be good anti-apoptotic strategies to protect cells from death [102,103].

In $\mathrm{P} 23 \mathrm{H}$ rats, it has also been demonstrated that mTOR/Akt and autophagy signaling, along with the expression of Bcl-2 family proteins, is altered during adRP progression, and this correlated with $\mathrm{Ca}^{2+}$ changes promoted by calpain and caspase 12 activation. ROS accumulation may also induce mitochondrial and lysosomal membrane permeabilization, releasing pro-apoptotic proteins [23,104-107].

The exact mechanisms by which safranal and TUDCA exert neuroprotection remain unclear. Our hypotheses concerning the possible pathways in which these compounds could act in order to counterbalance apoptosis in the $\mathrm{P} 23 \mathrm{H}$ retina are schematized in Figure 3. 



Figure 3. Apoptotic pathways in the retina. Schematic representation of the most relevant pathways involved in programmed cell death (PCD) in the $\mathrm{P} 23 \mathrm{H}$ rat retina and likely targets for safranal and TUDCA. Most retinal cells die as a result of caspase-dependent pathways, although caspase-independent pathways involving calpains and/or cathepsins are also present. TUDCA activity may be exerted by decreasing ER stress, stabilizing the outer mitochondrial membrane and blocking calpain-driven apoptosis. Among the major causes of stress and cell death in the retina is the accumulation of reactive oxygen species (ROS) associated with pathological conditions and damage to both mitochondria and lysosomes. Safranal could be decreasing oxidative stress due to ROS elevation, thereby ameliorating cell death. Akt/PKB: Protein kinase B; Bad: Bcl-2-associated agonist of cell death; Bax: Bcl-2-associated X protein; Bcl-2: apoptosis regulator Bcl-2 (B-cell lymphoma-2); ER: endoplasmic reticulum; MOMP: mitochondrial outer membrane permeabilization; PTPC: permeability transition pore complex; ROS: reactive oxygen species.

\subsection{Neuroprotective Mechanisms of Safranal}

Saffron and its constituents, such as safranal, have been shown to exert cytoprotective effects on a wide spectrum of tissues, including nervous tissue, in a dose-dependent manner [63]. The best described mechanism by which saffron acts against cell damage is its antioxidant activity (Figure 3), relying on its ability to scavenge free radicals [108]. Also, this compound promotes an increase of the natural antioxidant defenses of the cell [61], together with its capacity to protect DNA and RNA from harmful chemical reactions by forming ligand-polynucleotide complexes [109,110].

The retina is one of the tissues with the highest oxygen consumption in the body of mammals. Retinal degeneration is accompanied by an increase in ROS and an abnormal rise in cytosolic $\mathrm{Ca}^{2+}$, 
which are able to permeabilize the mitochondrial [102] and lysosomal membranes, thereby amplifying the pro-apoptotic events $[105,111]$. In this scenario, safranal could decrease the activation of different apoptotic pathways that may be stimulated by the increase in ROS (Figure 3). In addition to this high level of antioxidant activity, safranal has also been shown to be capable of downregulating Bax and caspase 3 expression in muscle cells of rats with myocardial ischemia-reperfusion injury [112], suggesting an additional antiapoptotic activity. All these results have contributed to initiate clinical trials aimed at testing the activity of saffron in patients with age-related macular degeneration [49] based on the success of previous studies in animal models [50,51].

\subsection{Neuroprotective Mechanisms of TUDCA}

The activity of TUDCA has been widely investigated in several models of neurodegenerative diseases. Previous works have demonstrated that TUDCA is able to modulate apoptosis by regulating different pro-apoptotic pathways $[113,114]$. This may be related to the neurotoxicity caused by accumulation of amyloid beta $\beta$ in cells, as it occurs in Alzheimer disease $[115,116]$. It has been observed that TUDCA inhibits $\beta$-induced mitochondrial permeabilization and cytocrome $\mathrm{c}$ release in primary rat neurons and astrocyte cultures [117] and is able to stabilize the mitochondrial membrane, thereby preventing Bax translocation and MOMP formation in isolated mitochondria [118]. In addition, TUDCA has been described as a chemical chaperone capable of reducing the ER stress caused by misfolded proteins in diabetic retinopathy models $[119,120]$ and in cases of retinal degeneration involving ER stress [72,121]. TUDCA protects against ER stress presumably by modulating intracellular $\mathrm{Ca}^{2+}$ levels and thereby inhibiting calpains and caspase 12 activation (Figure 3) [119,122].

All these features make TUDCA a good therapeutic agent, due to its effectiveness not only in delaying photoreceptor cell loss, but also in slowing the degeneration of inner retinal cells. Therefore, TUDCA might be useful in combination with antioxidants and anti-inflammatory agents, together with therapies based on gene therapy or the transplantation of new photoreceptors, to further ensure the success of the latter. Researchers are also currently focusing on different strategies to deliver this compound to the retina. Systemic administration of TUDCA requires high concentrations to be effective against retinal tissue neurodegeneration [41,65-72]. Although TUDCA is well tolerated at high doses, new delivery mechanisms are being explored in order to achieve effective concentrations of this drug in the retina without extensive spreading to other tissues. In this sense, administration by intravitreal injection of TUDCA-loaded microspheres seems to provide all the advantages of the treatment, while using concentrations of this compound lower than those used in systemic administration [123].

\section{Conclusions}

The present review shows the capacity of the natural compounds safranal and TUDCA to delay retinal degeneration. Both safranal and TUDCA were able to reduce rod and cone photoreceptor loss, as well as to protect their electroretinographic response in the $\mathrm{P} 23 \mathrm{H}$ adRP rat model. The synaptic contacts between photoreceptors and their postsynaptic cells were also more numerous and better preserved as compared to untreated animals in all experiments. The treatments were also able to diminish the degree of secondary remodeling that takes place in the retina after photoreceptor cell death, improving the condition of cells postsynaptic to photoreceptors. Moreover, we documented that TUDCA reduces 
the number and activation of microglial cells in the P23H model of RP. The neuroprotective effects of safranal and TUDCA make these natural compounds potentially useful for delaying retinal degeneration in retinal pathologies.

\section{Acknowledgments}

This research was supported by grants from the Spanish Ministry of Economy and CompetitivenessFEDER (BFU2012-36845), Instituto de Salud Carlos III (RETICS RD12/0034/0010), Asociación Retina Asturias, Fundación Jesús Gangoiti, Organización Nacional de Ciegos Españoles (ONCE) and FUNDALUCE.

\section{Author Contributions}

L.F.-S., P.L., and N.C. conceived and designed the work, interpreted the data, and drafted and revised the paper. L.F.-S, A.A. A.N. and V.M. acquired and analyzed the data, and drafted the paper. N.C. supervised the research.

\section{Conflicts of Interest}

The authors declare no conflict of interest.

\section{References}

1. Cuenca, N.; Fernandez-Sanchez, L.; Campello, L.; Maneu, V.; de la Villa, P.; Lax, P.; Pinilla, I. Cellular responses following retinal injuries and therapeutic approaches for neurodegenerative diseases. Prog. Retin. Eye Res. 2014, 43, 17-75.

2. Stone, J.; van Driel, D.; Valter, K.; Rees, S.; Provis, J. The locations of mitochondria in mammalian photoreceptors: Relation to retinal vasculature. Brain Res. 2008, 1189, 58-69.

3. Szikra, T.; Krizaj, D. Intracellular organelles and calcium homeostasis in rods and cones. Vis. Neurosci. 2007, 24, 733-743.

4. Panfoli, I.; Calzia, D.; Bianchini, P.; Ravera, S.; Diaspro, A.; Candiano, G.; Bachi, A.; Monticone, M.; Aluigi, M.G.; Barabino, S.; et al. Evidence for aerobic metabolism in retinal rod outer segment disks. Int. J. Biochem. Cell Biol. 2009, 41, 2555-2565.

5. Panfoli, I.; Calzia, D.; Ravera, S.; Morelli, A.M.; Traverso, C.E. Extra-mitochondrial aerobic metabolism in retinal rod outer segments: New perspectives in retinopathies. Med. Hypotheses 2012, 78, 423-427.

6. Johnson, J.E., Jr.; Perkins, G.A.; Giddabasappa, A.; Chaney, S.; Xiao, W.; White, A.D.; Brown, J.M.; Waggoner, J.; Ellisman, M.H.; Fox, D.A. Spatiotemporal regulation of ATP and $\mathrm{Ca}^{2+}$ dynamics in vertebrate rod and cone ribbon synapses. Mol. Vis. 2007, 13, 887-919.

7. Nicholls, D.G.; Johnson-Cadwell, L.; Vesce, S.; Jekabsons, M.; Yadava, N. Bioenergetics of mitochondria in cultured neurons and their role in glutamate excitotoxicity. J. Neurosci. Res. 2007, 85, 3206-3212. 
8. Yadava, N.; Nicholls, D.G. Spare respiratory capacity rather than oxidative stress regulates glutamate excitotoxicity after partial respiratory inhibition of mitochondrial complex I with rotenone. J. Neurosci. 2007, 27, 7310-7317.

9. Tomany, S.C.; Wang, J.J.; van Leeuwen, R.; Klein, R.; Mitchell, P.; Vingerling, J.R.; Klein, B.E.; Smith, W.; de Jong, P.T. Risk factors for incident age-related macular degeneration: Pooled findings from 3 continents. Ophthalmology 2004, 111, 1280-1287.

10. Khan, J.C.; Thurlby, D.A.; Shahid, H.; Clayton, D.G.; Yates, J.R.; Bradley, M.; Moore, A.T.; Bird, A.C. Smoking and age related macular degeneration: The number of pack years of cigarette smoking is a major determinant of risk for both geographic atrophy and choroidal neovascularisation. Br. J. Ophthalmol. 2006, 90, 75-80.

11. Sanvicens, N.; Gomez-Vicente, V.; Masip, I.; Messeguer, A.; Cotter, T.G. Oxidative stress-induced apoptosis in retinal photoreceptor cells is mediated by calpains and caspases and blocked by the oxygen radical scavenger CR-6. J. Biol. Chem. 2004, 279, 39268-39278.

12. Chrysostomou, V.; Rezania, F.; Trounce, I.A.; Crowston, J.G. Oxidative stress and mitochondrial dysfunction in glaucoma. Curr. Opin. Pharmacol. 2013, 13, 12-15.

13. Himori, N.; Yamamoto, K.; Maruyama, K.; Ryu, M.; Taguchi, K.; Yamamoto, M.; Nakazawa, T. Critical role of $\mathrm{Nrf} 2$ in oxidative stress-induced retinal ganglion cell death. J. Neurochem. 2013, 127, 669-680.

14. Kowluru, R.A.; Tang, J.; Kern, T.S. Abnormalities of retinal metabolism in diabetes and experimental galactosemia. VII. Effect of long-term administration of antioxidants on the development of retinopathy. Diabetes 2001, 50, 1938-1942.

15. Shanab, A.Y.; Nakazawa, T.; Ryu, M.; Tanaka, Y.; Himori, N.; Taguchi, K.; Yasuda, M.; Watanabe, R.; Takano, J.; Saido, T.; et al. Metabolic stress response implicated in diabetic retinopathy: The role of calpain, and the therapeutic impact of calpain inhibitor. Neurobiol. Dis. 2012, 48, 556-567.

16. Du, Y.; Miller, C.M.; Kern, T.S. Hyperglycemia increases mitochondrial superoxide in retina and retinal cells. Free Radic. Biol. Med. 2003, 35, 1491-1499.

17. Du, Y.; Cramer, M.; Lee, C.A.; Tang, J.; Muthusamy, A.; Antonetti, D.A.; Jin, H.; Palczewski, K.; Kern, T.S. Adrenergic and serotonin receptors affect retinal superoxide generation in diabetic mice: Relationship to capillary degeneration and permeability. FASEB J. 2015, 29, 2194-2204.

18. Muller, F.L.; Liu, Y.; van Remmen, H. Complex III releases superoxide to both sides of the inner mitochondrial membrane. J. Biol. Chem. 2004, 279, 49064-49073.

19. Murakami, Y.; Notomi, S.; Hisatomi, T.; Nakazawa, T.; Ishibashi, T.; Miller, J.W.; Vavvas, D.G. Photoreceptor cell death and rescue in retinal detachment and degenerations. Prog. Retin. Eye Res. 2013, 37, 114-140.

20. Strasser, A.; O’Connor, L.; Dixit, V.M. Apoptosis signaling. Annu. Rev. Biochem. 2000, 69, 217-245.

21. Kuan, C.Y.; Roth, K.A.; Flavell, R.A.; Rakic, P. Mechanisms of programmed cell death in the developing brain. Trends Neurosci. 2000, 23, 291-297.

22. Fong, W.G.; Tsilfidis, C. Retinal degeneration and cellular suicide. Adv. Exp. Med. Biol. 2012, 723, 207-214. 
23. Sancho-Pelluz, J.; Arango-Gonzalez, B.; Kustermann, S.; Romero, F.J.; van Veen, T.; Zrenner, E.; Ekstrom, P.; Paquet-Durand, F. Photoreceptor cell death mechanisms in inherited retinal degeneration. Mol. Neurobiol. 2008, 38, 253-269.

24. Arango-Gonzalez, B.; Trifunovic, D.; Sahaboglu, A.; Kranz, K.; Michalakis, S.; Farinelli, P.; Koch, S.; Koch, F.; Cottet, S.; Janssen-Bienhold, U.; et al. Identification of a common non-apoptotic cell death mechanism in hereditary retinal degeneration. PLOS ONE 2014, 9, e112142.

25. Kaur, J.; Mencl, S.; Sahaboglu, A.; Farinelli, P.; van Veen, T.; Zrenner, E.; Ekstrom, P.; Paquet-Durand, F.; Arango-Gonzalez, B. Calpain and PARP activation during photoreceptor cell death in $\mathrm{P} 23 \mathrm{H}$ and S334ter rhodopsin mutant rats. PLoS ONE 2011, 6, e22181.

26. Wong, P. Apoptosis, retinitis pigmentosa, and degeneration. Biochem. Cell Biol. 1994, 72, 489-498.

27. Portera-Cailliau, C.; Sung, C.H.; Nathans, J.; Adler, R. Apoptotic photoreceptor cell death in mouse models of retinitis pigmentosa. Proc. Natl. Acad. Sci. USA 1994, 91, 974-978.

28. Doonan, F.; Donovan, M.; Cotter, T.G. Activation of multiple pathways during photoreceptor apoptosis in the rd mouse. Investig. Ophthalmol. Vis. Sci. 2005, 46, 3530-3538.

29. Daiger, S. P. RetNet. Available online: https://sph.uth.edu/retnet/disease.htm (accessed on 28 July 2015).

30. Dryja, T.P.; McGee, T.L.; Reichel, E.; Hahn, L.B.; Cowley, G.S.; Yandell, D.W.; Sandberg, M.A.; Berson, E.L. A point mutation of the rhodopsin gene in one form of retinitis pigmentosa. Nature 1990, 343, 364-366.

31. Dryja, T.P.; McEvoy, J.A.; McGee, T.L.; Berson, E.L. Novel rhodopsin mutations Gly114Val and Gln184Pro in dominant retinitis pigmentosa. Investig. Ophthalmol. Vis. Sci. 2000, 41, 3124-3127.

32. Kaushal, S.; Khorana, H.G. Structure and function in rhodopsin. 7. Point mutations associated with autosomal dominant retinitis pigmentosa. Biochemistry 1994, 33, 6121-6128.

33. Illing, M.E.; Rajan, R.S.; Bence, N.F.; Kopito, R.R. A rhodopsin mutant linked to autosomal dominant retinitis pigmentosa is prone to aggregate and interacts with the ubiquitin proteasome system. J. Biol. Chem. 2002, 277, 34150-34160.

34. Reme, C.E.; Grimm, C.; Hafezi, F.; Marti, A.; Wenzel, A. Apoptotic cell death in retinal degenerations. Prog. Retin. Eye Res. 1998, 17, 443-464.

35. Machida, S.; Kondo, M.; Jamison, J.A.; Khan, N.W.; Kononen, L.T.; Sugawara, T.; Bush, R.A.; Sieving, P.A. P23H rhodopsin transgenic rat: correlation of retinal function with histopathology. Investig. Ophthalmol. Vis. Sci. 2000, 41, 3200-3209.

36. Berson, E.L.; Rosner, B.; Sandberg, M.A.; Dryja, T.P. Ocular findings in patients with autosomal dominant retinitis pigmentosa and a rhodopsin gene defect (Pro-23-His). Arch. Ophthalmol. 1991, 109, 92-101.

37. Cuenca, N.; Pinilla, I.; Sauve, Y.; Lu, B.; Wang, S.; Lund, R.D. Regressive and reactive changes in the connectivity patterns of rod and cone pathways of $\mathrm{P} 23 \mathrm{H}$ transgenic rat retina. Neuroscience 2004, 127, 301-317.

38. Garcia-Ayuso, D.; Salinas-Navarro, M.; Agudo, M.; Cuenca, N.; Pinilla, I.; Vidal-Sanz, M.; Villegas-Perez, M.P. Retinal ganglion cell numbers and delayed retinal ganglion cell death in the $\mathrm{P} 23 \mathrm{H}$ rat retina. Exp. Eye Res. 2010, 91, 800-810.

39. Kolomiets, B.; Dubus, E.; Simonutti, M.; Rosolen, S.; Sahel, J.A.; Picaud, S. Late histological and functional changes in the $\mathrm{P} 23 \mathrm{H}$ rat retina after photoreceptor loss. Neurobiol. Dis. 2010, 38, 47-58. 
40. Esquiva, G.; Lax, P.; Cuenca, N. Impairment of intrinsically photosensitive retinal ganglion cells associated with late stages of retinal degeneration. Investig. Ophthalmol. Vis. Sci. 2013, 54, 4605-4618.

41. Fernandez-Sanchez, L.; Lax, P.; Pinilla, I.; Martin-Nieto, J.; Cuenca, N. Tauroursodeoxycholic acid prevents retinal degeneration in transgenic P23H rats. Investig. Ophthalmol. Vis. Sci.2011, 52, 4998-5008.

42. Fernandez-Sanchez, L.; Lax, P.; Esquiva, G.; Martin-Nieto, J.; Pinilla, I.; Cuenca, N. Safranal, a saffron constituent, attenuates retinal degeneration in $\mathrm{P} 23 \mathrm{H}$ rats. PLOS ONE 2012, 7, e43074.

43. Fernandez-Sanchez, L.; Lax, P.; Isiegas, C.; Ayuso, E.; Ruiz, J.M.; de la Villa, P.; Bosch, F.; de la Rosa, E.J.; Cuenca, N. Proinsulin slows retinal degeneration and vision loss in the P23H rat model of retinitis pigmentosa. Hum. Gene Ther. 2012, 23, 1290-1300.

44. Lax, P.; Esquiva, G.; Altavilla, C.; Cuenca, N. Neuroprotective effects of the cannabinoid agonist HU210 on retinal degeneration. Exp. Eye Res. 2014, 120, 175-185.

45. Javadi, B.; Sahebkar, A.; Emami, S.A. A survey on saffron in major islamic traditional medicine books. Iran. J. Basic Med. Sci. 2013, 16, 1-11.

46. Hosseinzadeh, H.; Nassiri-Asl, M. Avicenna's (Ibn Sina) the Canon of Medicine and saffron (Crocus sativus): A review. Phytother. Res. 2013, 27, 475-483.

47. Abdullaev, F.I. Biological effects of saffron. Biofactors 1993, 4, 83-86.

48. Bolhassani, A.; Khavari, A.; Bathaie, S.Z. Saffron and natural carotenoids: Biochemical activities and anti-tumor effects. Biochim. Biophys. Acta 2014, 1845, 20-30.

49. Bisti, S.; Maccarone, R.; Falsini, B. Saffron and retina: Neuroprotection and pharmacokinetics. Vis. Neurosci. 2014, 31, 355-361.

50. Falsini, B.; Piccardi, M.; Minnella, A.; Savastano, C.; Capoluongo, E.; Fadda, A.; Balestrazzi, E.; Maccarone, R.; Bisti, S. Influence of saffron supplementation on retinal flicker sensitivity in early age-related macular degeneration. Investig. Ophthalmol. Vis. Sci. 2010, 51, 6118-6124.

51. Maccarone, R.; di Marco, S.; Bisti, S. Saffron supplement maintains morphology and function after exposure to damaging light in mammalian retina. Investig. Ophthalmol. Vis. Sci. 2008, 49, 1254-1261.

52. Natoli, R.; Zhu, Y.; Valter, K.; Bisti, S.; Eells, J.; Stone, J. Gene and noncoding RNA regulation underlying photoreceptor protection: microarray study of dietary antioxidant saffron and photobiomodulation in rat retina. Mol. Vis. 2010, 16, 1801-1822.

53. Marco, F.D.; Romeo, S.; Nandasena, C.; Purushothuman, S.; Adams, C.; Bisti, S.; Stone, J. The time course of action of two neuroprotectants, dietary saffron and photobiomodulation, assessed in the rat retina. Am. J. Neurodegener. Dis. 2013, 2, 208-220.

54. Li, N.; Lin, G.; Kwan, Y.W.; Min, Z.D. Simultaneous quantification of five major biologically active ingredients of saffron by high-performance liquid chromatography. J. Chromatogr. A 1999, 849, 349-355.

55. Yamauchi, M.; Tsuruma, K.; Imai, S.; Nakanishi, T.; Umigai, N.; Shimazawa, M.; Hara, H. Crocetin prevents retinal degeneration induced by oxidative and endoplasmic reticulum stresses via inhibition of caspase activity. Eur. J. Pharmacol. 2011, 650, 110-119. 
56. Ishizuka, F.; Shimazawa, M.; Umigai, N.; Ogishima, H.; Nakamura, S.; Tsuruma, K.; Hara, H. Crocetin, a carotenoid derivative, inhibits retinal ischemic damage in mice. Eur. J. Pharmacol. 2013, 703, 1-10.

57. Ohno, Y.; Nakanishi, T.; Umigai, N.; Tsuruma, K.; Shimazawa, M.; Hara, H. Oral administration of crocetin prevents inner retinal damage induced by $N$-methyl-D-aspartate in mice. Eur. J. Pharmacol. 2012, 690, 84-89.

58. Laabich, A.; Vissvesvaran, G.P.; Lieu, K.L.; Murata, K.; McGinn, T.E.; Manmoto, C.C.; Sinclair, J.R.; Karliga, I.; Leung, D.W.; Fawzi, A.; et al. Protective effect of crocin against blue light- and white light-mediated photoreceptor cell death in bovine and primate retinal primary cell culture. Investig. Ophthalmol. Vis. Sci. 2006, 47, 3156-3163.

59. Xuan, B.; Zhou, Y.H.; Li, N.; Min, Z.D.; Chiou, G.C. Effects of crocin analogs on ocular blood flow and retinal function. J. Ocul. Pharmacol. Ther. 1999, 15, 143-152.

60. Qi, Y.; Chen, L.; Zhang, L.; Liu, W.B.; Chen, X.Y.; Yang, X.G. Crocin prevents retinal ischaemia/reperfusion injury-induced apoptosis in retinal ganglion cells through the PI3K/AKT signalling pathway. Exp. Eye Res. 2013, 107, 44-51.

61. Farahmand, S.K.; Samini, F.; Samini, M.; Samarghandian, S. Safranal ameliorates antioxidant enzymes and suppresses lipid peroxidation and nitric oxide formation in aged male rat liver. Biogerontology 2013, 14, 63-71.

62. Hosseinzadeh, H.; Modaghegh, M.H.; Saffari, Z. Crocus sativus L. (Saffron) extract and its active constituents (crocin and safranal) on ischemia-reperfusion in rat skeletal muscle. Evid. Based Complement. Altern. Med. 2009, 6, 343-350.

63. Hosseinzadeh, H.; Sadeghnia, H.R. Safranal, a constituent of Crocus sativus (saffron), attenuated cerebral ischemia induced oxidative damage in rat hippocampus. J. Pharm. Pharm. Sci. 2005, 8, 394-399.

64. Boatright, J.H.; Moring, A.G.; McElroy, C.; Phillips, M.J.; Do, V.T.; Chang, B.; Hawes, N.L.; Boyd, A.P.; Sidney, S.S.; Stewart, R.E.; et al. Tool from ancient pharmacopoeia prevents vision loss. Mol. Vis. 2006, 12, 1706-1714.

65. Phillips, M.J.; Walker, T.A.; Choi, H.Y.; Faulkner, A.E.; Kim, M.K.; Sidney, S.S.; Boyd, A.P.; Nickerson, J.M.; Boatright, J.H.; Pardue, M.T. Tauroursodeoxycholic acid preservation of photoreceptor structure and function in the rd10 mouse through postnatal day 30 . Investig. Ophthalmol. Vis. Sci. 2008, 49, 2148-2155.

66. Boatright, J.H.; Nickerson, J.M.; Moring, A.G.; Pardue, M.T. Bile acids in treatment of ocular disease. J. Ocul. Biol. Dis. Infor. 2009, 2, 149-159.

67. Oveson, B.C.; Iwase, T.; Hackett, S.F.; Lee, S.Y.; Usui, S.; Sedlak, T.W.; Snyder, S.H.; Campochiaro, P.A.; Sung, J.U. Constituents of bile, bilirubin and TUDCA, protect against oxidative stress-induced retinal degeneration. J. Neurochem. 2011, 116, 144-153.

68. Drack, A.V.; Dumitrescu, A.V.; Bhattarai, S.; Gratie, D.; Stone, E.M.; Mullins, R.; Sheffield, V.C. TUDCA slows retinal degeneration in two different mouse models of retinitis pigmentosa and prevents obesity in Bardet-Biedl syndrome type 1 mice. Investig. Ophthalmol. Vis. Sci. 2012, 53, $100-106$. 
69. Woo, S.J.; Kim, J.H.; Yu, H.G. Ursodeoxycholic acid and tauroursodeoxycholic acid suppress choroidal neovascularization in a laser-treated rat model. J. Ocul. Pharmacol. Ther. 2010, 26, 223-229.

70. Mantopoulos, D.; Murakami, Y.; Comander, J.; Thanos, A.; Roh, M.; Miller, J.W.; Vavvas, D.G. Tauroursodeoxycholic acid (TUDCA) protects photoreceptors from cell death after experimental retinal detachment. PLoS ONE 2011, 6, e24245.

71. Gaspar, J.M.; Martins, A.; Cruz, R.; Rodrigues, C.M.; Ambrosio, A.F.; Santiago, A.R. Tauroursodeoxycholic acid protects retinal neural cells from cell death induced by prolonged exposure to elevated glucose. Neuroscience 2013, 253, 380-388.

72. Zhang, T.; Baehr, W.; Fu, Y. Chemical chaperone TUDCA preserves cone photoreceptors in a mouse model of Leber congenital amaurosis. Investig. Ophthalmol. Vis. Sci. 2012, 53, 3349-3356.

73. Langmann, T. Microglia activation in retinal degeneration. J. Leukoc. Biol. 2007, 81, 1345-1351.

74. Noailles, A.; Fernandez-Sanchez, L.; Lax, P.; Cuenca, N. Microglia activation in a model of retinal degeneration and TUDCA neuroprotective effects. J. Neuroinflamm. 2014, 11, doi:10.1186/s12974-014-0186-3.

75. Polazzi, E.; Monti, B. Microglia and neuroprotection: From in vitro studies to therapeutic applications. Prog. Neurobiol. 2010, 92, 293-315.

76. Cunningham, C. Microglia and neurodegeneration: The role of systemic inflammation. Glia 2013, 61, 71-90.

77. Reus, G.Z.; Fries, G.R.; Stertz, L.; Badawy, M.; Passos, I.C.; Barichello, T.; Kapczinski, F.; Quevedo, J. The role of inflammation and microglial activation in the pathophysiology of psychiatric disorders. Neuroscience 2015, 300, 141-154.

78. Jebelli, J.; Su, W.; Hopkins, S.; Pocock, J.; Garden, G.A. Glia: Guardians, gluttons, or guides for the maintenance of neuronal connectivity? Ann. N. Y. Acad. Sci. 2015, doi:10.1111/nyas.12711.

79. Sanchez-Guajardo, V.; Tentillier, N.; Romero-Ramos, M. The relation between alpha-synuclein and microglia in Parkinson's disease: Recent developments. Neuroscience 2015, doi:10.1016/j.neuroscience.2015.02.008.

80. Lam, T.T.; Kwong, J.M.; Tso, M.O. Early glial responses after acute elevated intraocular pressure in rats. Investig. Ophthalmol. Vis. Sci. 2003, 44, 638-645.

81. Bosco, A.; Steele, M.R.; Vetter, M.L. Early microglia activation in a mouse model of chronic glaucoma. J. Comp. Neurol. 2011, 519, 599-620.

82. Penfold, P.L.; Madigan, M.C.; Gillies, M.C.; Provis, J.M. Immunological and aetiological aspects of macular degeneration. Prog. Retin. Eye Res. 2001, 20, 385-414.

83. Buschini, E.; Piras, A.; Nuzzi, R.; Vercelli, A. Age related macular degeneration and drusen: Neuroinflammation in the retina. Prog. Neurobiol. 2011, 95, 14-25.

84. Ng, T.F.; Streilein, J.W. Light-induced migration of retinal microglia into the subretinal space. Investig. Ophthalmol. Vis. Sci. 2001, 42, 3301-3310.

85. Zhang, C.; Shen, J.K.; Lam, T.T.; Zeng, H.Y.; Chiang, S.K.; Yang, F.; Tso, M.O. Activation of microglia and chemokines in light-induced retinal degeneration. Mol. Vis. 2005, 11, 887-895.

86. Gupta, N.; Brown, K.E.; Milam, A.H. Activated microglia in human retinitis pigmentosa, late-onset retinal degeneration, and age-related macular degeneration. Exp. Eye Res. 2003, 76, 463-471. 
87. Yoshida, N.; Ikeda, Y.; Notomi, S.; Ishikawa, K.; Murakami, Y.; Hisatomi, T.; Enaida, H.; Ishibashi, T. Laboratory evidence of sustained chronic inflammatory reaction in retinitis pigmentosa. Ophthalmology 2013, 120, e5-e12.

88. Peng, B.; Xiao, J.; Wang, K.; So, K.F.; Tipoe, G.L.; Lin, B. Suppression of microglial activation is neuroprotective in a mouse model of human retinitis pigmentosa. J. Neurosci. 2014, 34, 8139-8150.

89. Yang, P.; de Vos, A.F.; Kijlstra, A. Macrophages in the retina of normal Lewis rats and their dynamics after injection of lipopolysaccharide. Investig. Ophthalmol. Vis. Sci. 1996, 37, 77-85.

90. Yanguas-Casas, N.; Barreda-Manso, M.A.; Nieto-Sampedro, M.; Romero-Ramirez, L. Tauroursodeoxycholic acid reduces glial cell activation in an animal model of acute neuroinflammation. J. Neuroinflamm. 2014, 11, doi:10.1186/1742-2094-11-50.

91. Park, J.; Choi, H.; Min, J.S.; Park, S.J.; Kim, J.H.; Park, H.J.; Kim, B.; Chae, J.I.; Yim, M.; Lee, D.S. Mitochondrial dynamics modulate the expression of pro-inflammatory mediators in microglial cells. J. Neurochem. 2013, 127, 221-232.

92. Yang, L.P.; Wu, L.M.; Guo, X.J.; Tso, M.O.M. Activation of endoplasmic reticulum stress in degenerating photoreceptors of the rd1 mouse. Investig. Ophthalmol. Vis. Sci. 2007, 48, 5191-5198.

93. Sizova, O.S.; Shinde, V.M.; Lenox, A.R.; Gorbatyuk, M.S. Modulation of cellular signaling pathways in P23H rhodopsin photoreceptors. Cell. Signal. 2014, 26, 665-672.

94. Sanges, D.; Marigo, V. Cross-talk between two apoptotic pathways activated by endoplasmic reticulum stress: Differential contribution of caspase-12 and AIF. Apoptosis 2006, 11, 1629-1641.

95. Suzuki, K.; Hata, S.; Kawabata, Y.; Sorimachi, H. Structure, activation, and biology of calpain. Diabetes 2004, 53 (Suppl. 1), S12-S18.

96. Nguyen, A.T. H.; Campbell, M.; Kenna, P.F.; Kiang, A.S.; Tam, L.; Humphries, M.M.; Humphries, P. Calpain and Photoreceptor Apoptosis. Retin. Degener. Dis. 2012, 723, 547-552.

97. Tan, Y.; Dourdin, N.; Wu, C.; Veyra, T.D.; Elce, J.S.; Peter, A.; Greer, P.A. Ubiquitous calpains promote caspase-12 and JNK activation during endoplasmic reticulum stress-induced apoptosis. J. Biol. Chem. 2006, 281, 16016-16024.

98. Paquet-Durand, F.; Azadi, S.; Hauck, S.M.; Ueffing, M.; van Veen, T.; Ekstrom, P. Calpain is activated in degenerating photoreceptors in the rd1 mouse. $J$. Neurochem. 2006, 96, 802-814.

99. Ozaki, T.; Nakazawa, M.; Yamashita, T.; Sorimachi, H.; Hata, S.; Tomita, H.; Isago, H.; Baba, A.; Ishiguro, S.I. Intravitreal injection or topical eye-drop application of a $\mu$-calpain $\mathrm{C} 2 \mathrm{~L}$ domain peptide protects against photoreceptor cell death in Royal College of Surgeons' rats, a model of retinitis pigmentosa. Biochim. Biophys. Acta 2012, 1822, 1783-1795.

100. Keeble, J.A.; Gilmore, A.P. Apoptosis commitment-Translating survival signals into decisions on mitochondria. Cell Res. 2007, 17, 976-984.

101. Kunte, M.M.; Choudhury, S.; Manheim, J.F.; Shinde, V.M.; Miura, M.; Chiodo, V.A.; Hauswirth, W.W.; Gorbatyuk, O.S.; Gorbatyuk, M.S. ER stress is involved in T17M rhodopsin-induced retinal degeneration. Investig. Ophthalmol. Vis. Sci. 2012, 53, 3792-3800.

102. Garrido, C.; Galluzzi, L.; Brunet, M.; Puig, P.E.; Didelot, C.; Kroemer, G. Mechanisms of cytochrome c release from mitochondria. Cell Death Diff. 2006, 13, 1423-1433.

103. Tait, S.W.G.; Green, D.R. Mitochondria and cell death: Outer membrane permeabilization and beyond. Nat. Rev. Mol. Cell Biol. 2010, 11, 621-632. 
104. Sanvicens, N.; Cotter, T.G. Ceramide is the key mediator of oxidative stress-induced apoptosis in retinal photoreceptor cells. J. Neurochem. 2006, 98, 1432-1444.

105. Boya, P.; Kroemer, G. Lysosomal membrane permeabilization in cell death. Oncogene 2008, 27, 6434-6451.

106. Chahory, S.; Keller, N.; Martin, E.; Omri, B.; Crisanti, P.; Torriglia, A. Light induced retinal degeneration activates a caspase-independent pathway involving cathepsin D. Neurochem. Int. 2010, 57, 278-287.

107. Metrailler, S.; Schorderet, D.F.; Cottet, S. Early apoptosis of rod photoreceptors in Rpe $65^{-/-}$mice is associated with the upregulated expression of lysosomal-mediated autophagic genes. Exp. Eye Res. 2012, 96, 70-81.

108. Assimopoulou, N.; Sinakos, Z.; Papageorgiou, V.P. Radical scavenging activity of Crocus sativus L. extract and its bioactive constituents. Phytother. Res. 2005, 19, 997-1000.

109. Kanakis, C.D.; Tarantilis, P.A.; Tajmir-Riahi, H.A.; Polissiou, M.G. DNA interaction with saffron's secondary metabolites safranal, crocetin, and dimethylcrocetin. DNA Cell Biol. 2007, 26, 63-70.

110. Kanakis, C.D.; Tarantilis, P.A.; Pappas, C.; Bariyanga, J.; Tajmir-Riahi, H.A.; Polissiou, M.G. An overview of structural features of DNA and RNA complexes with saffron compounds: Models and antioxidant activity. J. Photochem. Photobiol. B 2009, 95, 204-212.

111. Kirkegaard, T.; Jaattela, M. Lysosomal involvement in cell death and cancer. Biochim. Biophys. Acta 2009, 1793, 746-754.

112. Bharti, S.; Golechha, M.; Kumari, S.; Siddiqui, K.M.; Arya, D.S. Akt/GSK-3beta/eNOS phosphorylation arbitrates safranal-induced myocardial protection against ischemia-reperfusion injury in rats. Eur. J. Nutr. 2012, 51, 719-727.

113. Ramalho, R.M.; Viana, R.J. S.; Low, W.C.; Steer, C.J.; Rodrigues, C.M. P. Bile acids and apoptosis modulation: An emerging role in experimental Alzheimer's disease. Trends Mol. Med. 2008, 14, 54-62.

114. Amaral, J.D.; Viana, R.J. S.; Ramalho, R.M.; Steer, C.J.; Rodrigues, C.M. P. Bile acids: Regulation of apoptosis by ursodeoxycholic acid. J. Lipid Res. 2009, 50, 1721-1734.

115. Nunes, A.F.; Amaral, J.D.; Lo, A.C.; Fonseca, M.B.; Viana, R.J.; Callaerts-Vegh, Z.; D’Hooge, R.; Rodrigues, C.M. TUDCA, a bile acid, attenuates amyloid precursor protein processing and amyloid-beta deposition in APP/PS1 mice. Mol. Neurobiol. 2012, 45, 440-454.

116. Sola, S.; Castro, R.E.; Laires, P.A.; Steer, C.J.; Rodrigues, C.M. Tauroursodeoxycholic acid prevents amyloid-beta peptide-induced neuronal death via a phosphatidylinositol 3-kinase-dependent signaling pathway. Mol. Med. 2003, 9, 226-234.

117. Rodrigues, C.M.; Solá, S.; Silva, R.; Brites, D. Bilirubin and amyloid-beta peptide induce cytochrome c release through mitochondrial membrane permeabilization. Mol. Med. 2000, 6, 936-946.

118. Rodrigues, C.M.; Sola, S.; Sharpe, J.C.; Moura, J.J.; Steer, C.J. Tauroursodeoxycholic acid prevents Bax-induced membrane perturbation and cytochrome $\mathrm{C}$ release in isolated mitochondria. Biochemistry 2003, 42, 3070-3080.

119. Ozcan, L.; Ergin, A.S.; Lu, A.; Chung, J.; Sarkar, S.; Nie, D.; Myers, M.G.; Ozcan, U. Endoplasmic reticulum stress plays a central role in development of leptin resistance. Cell Metab. 2009, 9, 35-51. 
120. Malo, A.; Krüger, B.; Seyhun, E.; Schäfer, C.; Hoffmann, R.T.; Göke, B.; Kubisch, C.H. Tauroursodeoxycholic acid reduces endoplasmic reticulum stress, trypsin activation, and acinar cell apoptosis while increasing secretion in rat pancreatic acini. Am. J. Physiol. 2010, 299, G877-G886.

121. Duricka, D.L.; Brown, R.L.; Varnum, M.D. Defective trafficking of cone photoreceptor CNG channels induces the unfolded protein response and ER-stress-associated cell death. Biochem. J. 2012, 441, 685-696.

122. Xie, Q.; Khaoustov, V.I.; Chung, C.C.; Sohn, J.; Krishnan, B.; Lewis, D.E.; Yoffe, B. Effect of tauroursodeoxycholic acid on endoplasmic reticulum stress-induced caspase-12 activation. Hepatology 2002, 36, 592-601.

123. Herrero-Vanrell, R.; Cardillo, J.A.; Kuppermann, B.D. Clinical applications of the sustained-release dexamethasone implant for treatment of macular edema. Clin. Ophthalmol. 2011, 5, 139-146.

(C) 2015 by the authors; licensee MDPI, Basel, Switzerland. This article is an open access article distributed under the terms and conditions of the Creative Commons Attribution license (http://creativecommons.org/licenses/by/4.0/). 